\title{
HUMANITIES
}

Timing:

\section{A key concept for both karateka (空手家) and emergency physicians}

\author{
Clarissa Brisseau, BSc, MSc' Constance LeBlanc, MD, FCFP, CCFP(EM), MAEd ${ }^{2}$ \\ I. Class of 2022, Faculty of Medicine, Dalhousie University \\ 2. Professor, Department of Emergency Medicine, Dalhousie University
}

\begin{abstract}
The importance of skills learned through extracurricular activities to medical education, including both athletics and the arts, is undeniable. In this reflective article we draw parallels between the skills learned through the practice of karate, specifically sparring, and those required to perform a patient-centered medical interview in the Emergency Department. Although a collaborative style is traditionally used for both undertakings, there are often good reasons to vary the timing and approach. A high level of situational awareness is necessary to perform either of these tasks effectively.
\end{abstract}

$\mathrm{T}$ he most important part of the clinical encounter is inarguably the medical interview ${ }^{1,2,3,4}$. Not only does it provide essential information necessary to develop and refine a differential diagnosis, it also lays the foundation on which physicians build rapport and trust with their patients. The art of the medical interview, however, takes time to master as it requires balance between eliciting the information needed to inform diagnosis or treatment, soliciting clarification, providing redirection, and allowing time for the patient speak and be heard. Mismatches between the doctor's communication skills or style and the patient's needs leads to a strained doctor-patient relationship and negatively impacts the establishment of an effective therapeutic alliance. This can lead to insufficient sharing of information, the patient feeling as if their concerns have not been heard or validated, or feeling that they have not received competent care. Awareness of different communication skills and styles together with the flexibility to use them when appropriate are key to a successful medical interview ${ }^{5,6,7}$.

Emergency Medicine (EM) provides a unique set of interview challenges that are not frequently encountered in other medical disciplines: some of the time, it is a fast-paced speciality, and at other times it requires detail and caution. Emergency Physicians (EP) are constantly pressed for time given the constant influx of patients and the scarcity of space and physicians in the Emergency Department (ED). This creates consistent competing demands for their time and attention. Despite the multiple demands of this speciality, there is an expectation of both timely and high-quality care, both of which require an excellent medical history and a strong therapeutic alliance. In providing care to patients with urgent and emergent health complaints, timely diagnosis and management are core considerations.

Through observation and experience during a first-year medical student elective in EM, the student realized that adaptability in the medical interview is an essential skill. Below, we reflect on some of the cases a first-year student and preceptor encountered, and how these required varying communication styles to be effective and to successfully connect with and care for patients.

Both student and preceptor are karatekas practitioners of karate - and noted the fact that the principles and training to excel in the medical interview are aligned with other areas of life, most notably karate ${ }^{8}$. Karate relies on non-verbal cues and adaptation of timing, in addition to strong technical skills for success. The interview styles outlined below align with the approaches to timing responses in karate sparring: sen-no-sen and its subsets $y u$-no sen and tai-no-sen use near simultaneous sparring responses, while go-no-sen responds once the attack has been initiated.

\section{The Dialogue-based Interview or Sen-no-sen}

The classic medical interview is collaborative in style. This interview style allows the patient to tell their story or voice their concerns while the physician interjects at natural breakpoints in the conversation to ask probing questions or perhaps to redirect the flow of information, thereby gaining all the information required to provide care. Though this dialectical interview will generally meet expectations for both patients and doctors, it is not always feasible. Every interview is slightly different because of patients' different needs with respect to levels of opportunity to talk in the interview in order for feel heard and understood.

In karate, the dialogue-based or collaborative style interview aligns with sen-no-sen, in which your response to your partner's technique is initiated at the same time as their attack. This aligns with a dialectical 
or collaborative style: the physician acquires the information they need from a story collected through a conversation with the patient.

\section{The Patient-driven Interview or Go-no-sen}

There are patients who present to the ED needing more time and consideration to delineate the diagnosis and provide excellent care, thus requiring more time in the medical interview. This can be due to a greater than average need in some patients to control the interview process, patients whose care is very complex requiring that a lot of information be collected to ascertain the issues, or other challenges such as a high level of anxiety, for example. In this patient-driven (or family-driven) style, the patient will tell their story with minimal, or ideally without, interruption from the physician until it is completed. Some examples of patient-driven interviews are cases of minor trauma where the patient has a solid grasp of all the data required or cases of delivery of unexpected bad news, such as a major or serious diagnosis being shared in the ED. Sometimes a physician can prepare for this prior to talking with a patient, but this is not generally the case in the ED setting. One such case experienced by the authors involved an interaction with a young woman presenting with abdominal pain, nausea and diarrhea. Although her symptoms were in keeping with a case of gastroenteritis, she revealed late in the interview that she had been sexually assaulted a few days prior to her current illness. This impacted her ability to cope with her condition and she needed reassurance that the two events were not related. We did not know this before interacting with her and once divulged, quickly adapted our interview style from a dialectical conversation about her abdominal pain and diarrhea to one that provided her with ample space to share her recent traumatic event, on her own terms, while we listened carefully. This refocused interview style allowed for greater empathy and exploration of concerns surrounding the assault and the care and support she would require after discharge from the ED. Though this conversation took considerably more than the anticipated time, spending the time to talk about these issues in a sensitive and emphatic way and making time for her to share her story allowed for greater connection and ultimately superior care. As a profession, doctors are not fantastic at giving patients sufficient opportunity to speak uninterrupted. It takes about seven seconds, on average, for a physician to interrupt their patients during a medical encounter ${ }^{9,10}$. The demands of EM provide even more pressure for a physician to direct the interview and gather the information they need as quickly as possible. In sensitive cases like this one, however, a shift in approach is indicated. The EP may be the first health care provider with whom a patient has raised an issue, or the patient may either fear or receive confirmation of a major health problem or a terminal illness. It is important to keep the patient and their family at the centre of our concern and give them the space they need to process and discuss their concerns. While this does take time, neglecting this facet of the caring relationship precludes our ability to provide excellent care for our patients. The case described above illustrates the importance of physicians' attention to timing in the medical interview.

This aligns with go-no-sen in karate, an approach which involves responding to an incoming attack after your partner has initiated their technique. This approach will often provide important information that will inform your response. This may feel slower, but it allows for a better understanding of your partner before your counterattack. The patient-driven interview style involves these same principles of allowing the other person to share their story before the physician interjects, allowing for a more complete understanding of the situation before responding. What differentiates the need for go-no-sen or sen-no-sen is situational awareness, specifically of your partner and of yourself. Like in medicine, karate requires careful observation of the other person, focusing on their needs, and planning for an interaction in order to be successful. However, flexibility and many hours of deliberate practice are necessary to achieve mastery in either.

The Physician-driven Interview or Tai-no-sen There are also patients who require a more physician-driven interview. Here, the physician drives the interview with more close-ended questions, the goal being rapid and accurate collection of factual information to inform care. Emergency Physicians will encounter multiple co-morbid patients where complexity may dictate a more closed approach as admission is inevitable, or patients with critical and emergent conditions who are predicted to be soon unable to respond and provide basic and critical information. This was encountered in an interaction with a patient with cellulitis of the leg. It was rapidly apparent that the key issue was the infection, but that this condition was impacted by factors including poverty, social isolation, and mental illness all intersecting to make a complex presentation. As this patient definitely required an admission to the hospital, time spent addressing these concerns acutely was not of benefit at the time of the ED visit, although would be impactful on her health and wellbeing over time. In order to initiate care and consult effectively, a narrow target on the infected leg 
was indicated and a more direct line of questioning was used for efficiency. Although this style does not allow ample opportunity for the patient to talk, this must be done with empathy and concern so that immediate changes to care to meet these individual needs can be initiated. It is important to not to push the conversation forward too quickly, as some tangents provide useful important insights into complex healthcare situations. In this case, sensitivity to these realities and attention to the social determinants of health remain important. Patients do not live in a sterile ED, and sometimes as a physician you can only deal with one problem and the characteristics and conditions that impact that condition directly. Another situation in which a very direct approach is indicated is that of the patient presenting with an emergent condition when critical information is required to inform urgent care decisions. An example of this is a patient with severe, acute respiratory distress when intubation is urgently indicated making very few questions feasible due to the time constraint. Key pieces of data are necessary to provide quality patient-centered care, including patient wishes, next of kin contact information and allergies. These must be obtained rapidly, making the use directed questioning appropriate. This communication style is far less satisfying for all involved parties.

In karate, Tai-no-sen, one subset of Sen-no-sen, involves responding to your partner's technique with an attack before they have had an opportunity to finish it. In a medical interview this could be an interjection for clarification or to collect greater detail in a facet of the interview. A second subset of sen-no-sen is $y u$-no-sen. This is where a karateka will shift their body position prior to an incoming attack in order to avoid it and to set up a counterattack. This approach aligns with a redirection of the information provided by a patient and is a very common technique in the medical interview. A physician-driven interview accomplishes some important tasks in the ED by asking specific and closed-ended questions before the patient can contribute these data themselves, because the interview is at risk of being derailed or worse, making necessary information unavailable.

\section{Discussion}

Dialectical, physician-driven, and patient-driven styles of interviewing all rely on the same basic skills: question structure, question flow, empathy, and non-verbal communication. What differentiates these styles is timing, follow-up or probing questions, and the EP's attitude and attention to ensuring that all information to make a diagnosis and provide care is gathered while making sure that the patient feels heard. Reading the patients' non-verbal cues and maintaining a flexible style to match that of the patient is a challenging skillset to acquire. While an actual interview will rely on a range of approaches and techniques, consideration of different potential styles of responding to and meeting a patient's needs during physician education will foster the development of different approaches to the medical interview that will augment the quality of care.

\section{Conclusion}

Emergency Medicine provides a unique set of challenges and demands that make a collaborative style interview impracticable some of the time. The constant stress of patient numbers, the acuity of ED presentations, and the competing demands in this setting all lead to a sense of urgency within the medical interview. Physicians, especially EPs, must remain sensitive and adaptable to remain patient-centered. This elective experience has highlighted the importance of reflection on extracurricular activities and clinical work to foster skillset co-development in both karate and EM. This was true for both preceptor and student. In interactive activities such as EM or karate, situational awareness (Zanshi or 残心) and perfect timing (Kanpekina taimingu or 完 璧なタイミング) are necessary to achieve excellence. In order to do this effectively and efficiently, skill development and attention to non-verbal cues are essential in both disciplines.

"Understand that the essence of martial arts is not the art itself, but what's hidden deep within yourself."

Gogen Yamaguchi

"... practice your communication skills so that when important occasions arise, you will have the gift, the style, the sharpness, the clarity, and the emotions to affect other people."11 Jim Rohn

\section{Acknowledgements}

We would like to acknowledge Sensei Tony Tam for his insightful input and support.

\section{References}

1. Hall J. A., Roter D. L., Rand C. S. Communication of affect between patient and physician. J Health Soc Behav. 1981;22(1):18-30.

2. Duffy F. D., Gordon G. H., Whelan G., et al. Assessing competence in communication and interpersonal skills: the Kalamazoo II report. Acad Med. 2004;79(6):495-507.

3. van Zanten M., Boulet J. R., McKinley D. W., DeChamplain A., Jobe A. C. Assessing the communication and interpersonal skills of graduates of international medical schools as part of the United States Medical Licensing Exam (USMLE) Step 2 Clinical Skills (CS) Exam. Acad Med. 2007;82(10 Suppl):S65-S68.

4. Brédart A., Bouleuc C., Dolbeault S. Doctor-patient 
